\title{
Acoustic Basis of Directional Acuity in Laboratory Mice
}

\author{
Amanda M. Lauer, Sean J. Slee, and Bradford J. May \\ Johns Hopkins University, Traylor Bldg., Room 521, 720 Rutland Avenue, Baltimore, MD 21205, USA
}

Received: 3 May 2011; Accepted: 15 June 2011; Online publication: 30 June 2011

\begin{abstract}
The acoustic basis of auditory spatial acuity was investigated in CBA/129 mice by relating patterns of behavioral errors to directional features of the headrelated transfer function (HRTF). Behavioral performance was assessed by training the mice to lick a water spout during sound presentations from a "safe" location and to suppress the response during presentations from "warning" locations. Minimum audible angles (MAAs) were determined by delivering the safe and warning sounds from different locations in the inter-aural horizontal and median vertical planes. HRTFs were measured at the same locations by implanting a miniature microphone and recording the gain of sound energy near the ear drum relative to free field. Mice produced an average MAA of $31^{\circ}$ when sound sources were located in the horizontal plane. Acoustic measures indicated that binaural inter-aural level differences (ILDs) and monaural spectral features of the HRTF change systematically with horizontal location and therefore may have contributed to the accuracy of behavioral performance. Subsequent manipulations of the auditory stimuli and the directional properties of the ear produced errors that suggest the mice primarily relied on ILD cues when discriminating changes in azimuth. The MAA increased beyond $80^{\circ}$ when the importance of ILD cues was minimized by testing in the median vertical plane. Although acoustic measures demonstrated a less robust effect of vertical location on spectral features of the HRTF, this poor performance provides further evidence for the insensitivity to spectral cues that was noted during behavioral testing in the horizontal plane.
\end{abstract}

Correspondence to: Bradford J. May · Johns Hopkins University . Traylor Bldg., Room 521, 720 Rutland Avenue, Baltimore, MD 21205, USA. Telephone: 410-614-1878; fax: 410-955-3162; email: bmay@jhu.edu
Keywords: spatial acuity, minimum audible angle, head-related transfer function, inter-aural level differences, spectral cues

\section{INTRODUCTION}

Despite the elaborate descriptions of the mechanisms of hearing and deafness that have been made possible by experiments in laboratory mice, there are relatively few descriptions of auditory perceptual behavior in this important species. In more comprehensively studied species such as the domestic cat, directional hearing has served as an ideal context for interpreting the acoustic cues and neural processes that support perception (Neff and Casseday 1977; Masterton 1997). At present, only three behavioral studies have characterized the perceptual limits of directional hearing in laboratory mice (Ehret and Dreyer 1984; Heffner et al. 2001; Allen and Ison 2010). These descriptions are restricted to the inter-aural horizontal plane, where the just detectable change in location between sound sources is reported to range from $7^{\circ}$ to $33^{\circ}$. The wide divergence of published data reflects a lack of common testing procedures, training strategies, and threshold criteria.

Similarly, there are only two published studies of the head-related transfer function (HRTF) of the mouse (Saunders and Garfinkle 1983; Chen et al. 1995). Both acoustic analyses used pure tones to measure the transformation of spectral energy from free field to the ear drum. Although tones provide sufficient resolution for characterizing inter-aural level differences (ILDs) at the lower range of mouse hearing, discrete frequency sampling is impractical for describing the fine spectral structure of the highfrequency HRTF. 
The present study was designed to extend our current knowledge of directional hearing in laboratory mice by combining a traditional psychophysical study with high-resolution HRTF measurements. Our behavioral method is similar to the conditioned avoidance paradigm that was used previously by Heffner et al. (2001), which allowed us to compare performance across two common strains of laboratory mice under similar testing conditions.

Initial experiments replicated previous measures of directional acuity in the inter-aural horizontal plane, where ILD cues are widely assumed to dictate the accuracy of behavioral responses in mice (Heffner et al. 2001; Allen and Ison 2010). Subsequent experiments isolated the relative importance of ILD and spectral information by manipulating the spectral content of auditory stimuli or the directional filtering properties of the HRTF. The final series of experiments analyzed the psychoacoustic basis of directional acuity in the median vertical plane, where directional acuity is assumed to reflect the processing of monaural spectral cues.

\section{METHODS}

All methods were reviewed annually and approved by the institutional animal care and use committee of The Johns Hopkins University School of Medicine.

\section{Behavioral subjects}

The discrimination of sound source location was measured in five adult CBA/129 mice. This hybrid strain was produced by crossing $\mathrm{CBA} / \mathrm{CaJ}$ and 129S6/ SvEvTac stains. All of the mice were female F1 hybrids. Our routine phenotyping procedures have confirmed that CBA/129 mice produce ABR thresholds, distortion product otoacoustic emissions, and acoustic startle reflexes that are indistinguishable from CBA/ $\mathrm{CaJ}$ mice, which are widely considered to be the "gold standard" of mouse hearing (Yoshida et al. 2000). Prior tests have also confirmed that this strain demonstrates normal patterns of hearing sensitivity and age-related hearing loss for laboratory mice. Each mouse was tested under multiple stimulus conditions at ages from 2 to 8 months. At these ages, CBA/129 mice are functionally mature but not old enough to be affected by age-related hearing loss.

The mice were given unlimited access to food during the 6 months of testing, but were waterdeprived to $80 \%$ of their free weight. On test days, the mice obtained a dilute mixture of juice and water as a reward for correct behavioral responses. On days when they were not tested, the mice were given access to hydrating gel. The mice remained healthy and active under the dietary regimen.

\section{Behavioral procedure}

The behavioral procedure was automated in MATLAB (MathWorks) using a microcomputer workstation (Hewlett Parkard, model xw4400). Contingencies of reinforcement were controlled through a multi-channel input/output processor (Tucker-Davis Technologies, model RX8). The testing system monitored behavioral responses, gated acoustic stimuli, and delivered juice rewards.

Testing was conducted inside a double-walled sound-attenuating chamber with inner dimensions of approximately $2 \times 2 \times 2 \mathrm{~m}$ (Industrial Acoustics Company). The interior surfaces of the chamber were lined with anechoic foam (Pinta Acoustic) to reduce acoustic reflections. Within the chamber, six speakers (Vifa XT25TG30-04) were mounted along a semicircular arc with a radius of $0.6 \mathrm{~m}$. A reference speaker was located at $0^{\circ}$ azimuth, $0^{\circ}$ elevation (directly in front of the subject). Five comparison speakers were positioned at angles of $20^{\circ}, 30^{\circ}, 50^{\circ}$, $70^{\circ}$, and $90^{\circ}$ relative to the reference speaker. The speaker array could be pivoted around the axis of the reference speaker to measure directional acuity in the inter-aural horizontal plane or median vertical plane. The speakers were matched in sound pressure level at frequencies between 0.5 and $50.0 \mathrm{kHz}$ to minimize potential loudness cues. The upper frequency limit of the test stimuli was dictated by the nominal $100-\mathrm{kHz}$ sampling rate of the digital sound generation system (Tucker-Davis Technologies, RX8).

The free-field response of each speaker is shown in Figure 1. These acoustic calibrations were made at the location of the subject's head using a Golay code method that is described later in the context of HRTF measurements (Zhou et al. 1992).

A mesh cage held the subject at the center of the speaker array. A syringe pump outside the chamber delivered juice to a tube on the forward facing wall of the cage. When the subject licked the tube, its head was directed toward the reference speaker. The subject's rate of licking was monitored with a contact sensor that was integrated with the spout.

Reinforcement contingencies of the behavioral task are summarized in Figure 2. During initial training, the reference speaker emitted an uninterrupted series of broadband noise bursts (240-ms on, 240-ms off, 10-ms rise/fall) and the spout delivered juice when contacted by the subject. These contingencies encouraged licking throughout initial training sessions of 10-15 min. Licking behavior was brought under stimulus control by gradually reducing the noise presentations to a twoburst sequence that occurred at randomly timed 


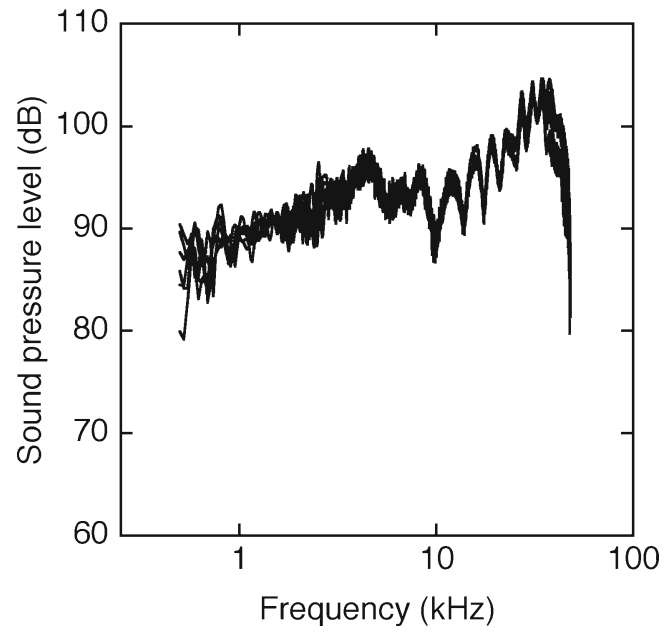

FIG. 1. Frequency response characteristics of the six speakers in the spatial array. Acoustic calibrations were recorded at the location of the subject's head, but without the subject in the testing apparatus. The sharp high-frequency cutoff near $50 \mathrm{kHz}$ is imposed by the sampling rate of the digital signal generator, not the speakers.

intervals (2-6 s). Juice was only available during the 720-ms interval of sound presentations.

During the final stages of training, the source of the noise bursts was varied to create two trial conditions. On safe trials, the reference speaker produced the bursts. On warning trials, a randomly selected comparison speaker served as the sound source. A shock generator on the spout was activated during the second burst of each warning trial. Mice learned to avoid the shocks by suppressing the licking behavior when they detected sound presentations from any of the comparison speakers. Safe trials $(p=$ $0.85)$ were presented more frequently than warning trials $(p=0.15)$ to maintain a high rate of licking. When the subject showed good discrimination of safe and warning trials, the length of the session was increased to approximately $60 \mathrm{~min}$.

The suppression of licking behavior on warning trials served as an index of the discriminability of the change in location between the reference and comparison speakers. The suppression effect was quantified by recording the presence of licks during the 400-ms time period that began $40 \mathrm{~ms}$ after the onset of the first safe or warning stimulus. Because the sampling interval terminated before the shock generator was activated, suppression was dictated by the discrimination of the comparison speaker location and not shock delivery.

The 400-ms sampling interval was divided into twenty 20-ms bins to determine the temporal characteristics of licking behavior. Each bin was scored with a 1 or 0 depending on whether or not the subject made contact with the spout. To verify a standardized rate of licking behavior at trial onset, the same binary sampling procedure was applied to the 400-ms interval leading up to the first presentation in the two-burst sequence.

Bin counts during stimulus presentations were converted to hit and false alarm rates to allow comparisons with previous descriptions of spatial acuity in other species. A response to a warning trial was designated a "hit" when the bin count was less than or equal to the numerical value that served as the subject's criterion for suppression. By the same convention, a response to a safe trial was designated a "false alarm." The criterion for suppression was adjusted for each subject to produce a false alarm rate near $16 \%$, which is typical for a well-trained subject in traditional operant procedures.

Discrimination performance was based on $d^{\prime}$. This method of signal detection analysis is relatively insensitive to changes in the criterion for suppression because it specifies the statistical separation of response percentiles on safe and warning trials. Setting a subject's criterion higher would increase the number of hits, but also the number of false alarms. Lowering the criterion would have the opposite effect. Once the criterion for suppression was established during testing with broadband noise in the horizontal plane, the same criterion was applied to all results from that subject to evaluate the effects of stimulus and pinna manipulations on both hit and false alarm rates.

The $d^{\prime}$ statistic was calculated in MATLAB as:

$$
d^{\prime}=\text { norminv (hit rate) }- \text { norminv (false alarm rate) } \text {. }
$$

The norminv function translates response percentiles to $z$ scores for a normal distribution. Conceptually, $d^{\prime}$ is the statistical separation of hit and false alarm rates in units of standard deviation.

By convention, the minimum audible angle (MAA) was defined as the change in location that produced $d^{\prime}=$ 1. Given an enforced false alarm rate near $16 \%, d^{\prime}=1$ when the hit rate approaches $50 \%$, which is another widely accepted definition of threshold in animal psychophysical studies.

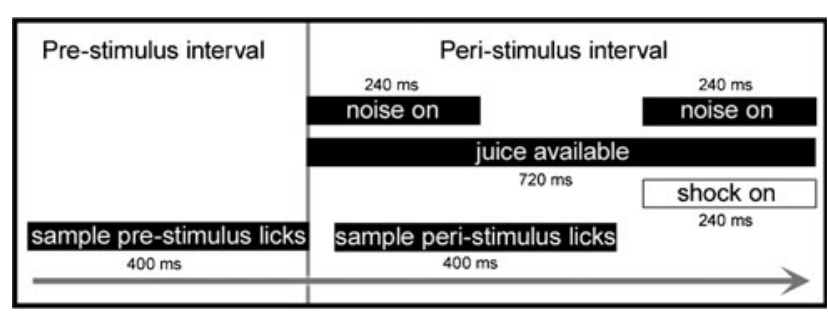

FIG. 2. Temporal relationship between stimulus presentations and behavioral sampling. The two noise bursts were presented from the reference speaker $\left(0^{\circ}\right.$ azimuth, $0^{\circ}$ elevation) on safe trials and from a randomly selected comparison speaker on warning trials. The shock was only activated during warning trials. 


\section{Stimulus manipulations}

Acoustic stimuli were synthesized online using digitalto-analog convertors with a nominal sampling rate of $100 \mathrm{kHz}$ (Tucker-Davis Technologies, RX8). Stimulus waveforms were amplified by a multi-channel power amplifier (Tucker-Davis Technologies, SA8) and passed independently to the speaker array. All stimuli were presented at a sensation level of approximately $40 \mathrm{~dB}$ SL based on the thresholds of auditory brainstem responses (ABRs). Sound levels were roved $\pm 2.5 \mathrm{~dB}$ to prevent the subjects from attending to directionally dependent loudness cues that may have been generated by room acoustics.

Initial experiments measured the limits of discrimination for source locations in the inter-aural horizontal plane. These tests were performed with broadband noise. Subsequent manipulations of the stimulus or the pinna were designed to isolate the acoustic cues that dictated the acuity of horizontal localization. The final series of experiments evaluated spatial acuity in the median vertical plane.

Specific protocols were as follows:

1. Broadband noise in the horizontal plane. Five mice were trained until performance showed no indication of further improvement and then tested for 711 sessions.

2. Band-limited noise in the horizontal plane. The frequency domain of the most effective localization cues was explored by measuring the discrimination of band-limited noise in the horizontal plane. The noise was either low-pass- or high-pass-filtered at a cutoff frequency of $10 \mathrm{kHz}$. The low-pass noise contained ILD effects, but not the monaural spectral cues that are created at higher frequencies by directional properties of the pinna. The highpass noise contained ILD effects that were enhanced in magnitude by the presence of spectral notches, as well as monaural spectral cues. Each condition was tested in five mice for one to two sessions.

3. Pure tones in the horizontal plane. ILD cues were mixed with spectral information during tests with high-frequency noise. The specific contribution of ILD information was addressed by measuring the MAA with tones. Frequencies were selected to represent multiple narrowband components of the low-pass noise ( 4 and $8 \mathrm{kHz}$ ) or high-pass noise (12 and $16 \mathrm{kHz}$ ). When pure tone localization proved to be a challenging task, response errors were reduced by limiting the discrimination to the $90^{\circ}$ speaker location. Each frequency was tested in five mice for one to three sessions.

4. Monaural ear plug. The directional acuity for broadband noise was examined while subjects were fitted with a monaural ear plug. ABR-based threshold measures indicated that the plug attenuated sounds in the ear canal by $15-20 \mathrm{~dB}$, creating an unnatural ILD for sound locations in the horizontal plane. This manipulation also altered spectral cues because the pinna was bonded (Vetbond, $3 \mathrm{M}$ ) in a folded position to hold the plug in place for several days.

Four mice were tested with either the near or far ear plugged. It was reasoned that the plug would degrade performance when placed in either ear if localization was based exclusively on binaural ILD cues. Ear plugging was performed under anesthesia, with tests beginning the following day. Four speaker locations $\left(30^{\circ}, 50^{\circ}, 70^{\circ}\right.$, and $\left.90^{\circ}\right)$ were sampled in one to two sessions.

5. Pinna distortion. Three mice had one pinna bonded in a folded position with no ear plug. This manipulation was designed to distort monaural spectral cues with less modification of ILD information. Consequently, if ILD processing was supplemented with monaural spectral cues, the distortion would induce performance deficits. Furthermore, if monaural cues were more reliable in one ear (e.g., the near ear), the deficit should be larger when the more informative ear was manipulated. Four speaker locations $\left(30^{\circ}, 50^{\circ}, 70^{\circ}\right.$, and $\left.90^{\circ}\right)$ were sampled in one to two sessions.

6. Broadband noise in the median plane. Given the assumption of symmetrical pinna acoustics, sound locations in the median plane do not generate directionally dependent binaural ILD effects. These tests, therefore, evaluated how well laboratory mice derived directional information when they were forced to rely on monaural spectral cues. Five mice were tested for two to four sessions.

\section{Acoustic measurements of the HRTF}

Acoustic measurements of the HRTF were made in two untrained adult mice from the CBA/129 strain. These experiments were conducted in the same apparatus and at the same speaker locations that were used to evaluate behavioral acuity. Methods for the acquisition and analysis of acoustic data have been described in detail in previous publications (Rice et al. 1992; Slee and Young 2010).

Briefly, the HRTF was characterized at 13 speaker locations in the horizontal plane and six locations in the median plane. The impulse response of each speaker location was generated by presenting pairs of 4,096-point Golay codes at a nominal sampling rate of $100 \mathrm{kHz}$ (Zhou et al. 1992). When converted to an analog signal, each code produced a 41-ms probe sound with a magnitude spectrum that varied $<1 \mathrm{~dB}$ at frequencies from 0 to $50 \mathrm{kHz}$. The impulse response 
was recorded with a miniature microphone (Knowles Electronics, model FG-23329-C05) and averaged over 19 presentations. Room reflections were removed by windowing the arrival time of the impulse response with a 400-point Hann function. The temporal waveform of the impulse response was transformed to a magnitude spectrum with a 4,096-point FFT, yielding a component resolution of $24 \mathrm{~Hz}$.

The free-field magnitude spectrum was measured by placing the microphone at the standardized head location of behavioral subjects, but without a mouse in the apparatus. The "in-ear" magnitude spectrum was measured from the same location, but the microphone was implanted in the ear canal of an anesthetized mouse. To replicate the effects of the head and torso on sound propagation, the mouse was arranged in the normal posture of a behaving subject.

Because the free-field response of the speaker array showed little variation between speakers or source locations (Fig. 1), the global free-field magnitude spectrum was determined by averaging the response across the 19 speaker/location combinations. HTRFs were derived by subtracting the global free-field magnitude spectrum from each in-ear magnitude spectrum. Thus, the HRTF summarizes the effects of pinna acoustics on sound energy in the ear canal, which is specified in terms of decibel gain increases (peaks) or decreases (notches) relative to free field.

\section{RESULTS}

\section{Directional acuity in the horizontal plane}

General patterns of lick suppression are summarized in Figure $3 \mathrm{~A}$ for presentations of broadband noise in the inter-aural horizontal plane. Results from each subject are based on an average of 2,556 safe trials $\left(0^{\circ}\right.$ azimuth) and 447 warning trials $\left(20-90^{\circ}\right.$ azimuth). The average pre-stimulus bin count ranged from 16.2 to 16.8 across the six speaker locations. These values approached the maximum bin count of 20 that was produced by continuous licking. A two-way ANOVA (implemented in MATLAB using the anova 2 function) indicated a statistically significant effect of subject on pre-stimulus bin counts $(p<0.05)$, which supports the use of individualized suppression criteria. Stable rates of licking were verified by the lack of a significant effect of location on pre-stimulus bin counts.

On average, licking activity during sound presentations progressively declined from a bin count of 17.3 at $0^{\circ}$ azimuth (safe trial) to a minimum of 5.5 at $90^{\circ}$ azimuth (the most lateralized warning trial). Both subject and location significantly influenced the rate of peri-stimulus licking (two-way ANOVA, $p<0.05$ ). These results confirm that the magnitude of suppres- sion is monotonically related to the discriminability of directional changes in the horizontal plane.

Each subject's criterion for suppression has been applied to the peri-stimulus bin counts in Figure 3A to
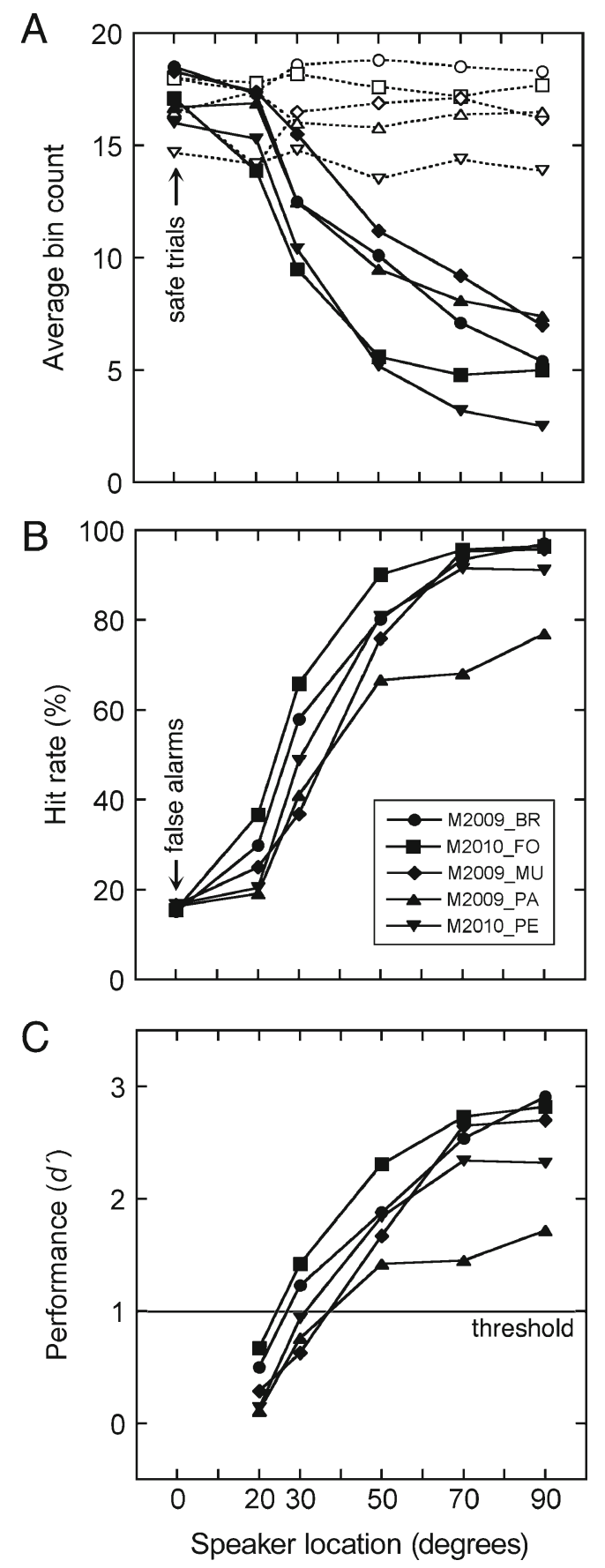

FIG. 3. Directional acuity for presentations of broadband noise in the inter-aural horizontal plane. A Licking behavior of six mice before stimulus presentations (dashed lines) and during stimulus presentations (solid lines). Bin counts indicate the presence of licking in 20 contiguous 20-ms intervals. B Psychometric functions relating hit and false alarm rates to the angular separation between safe and warning speakers. Response rates were calculated by applying individualized criterion scores to the bin counts in A. C Discrimination performance $\left(d^{\prime}\right)$ derived from the hit and false alarms rates in $\mathbf{B}$. 
generate the hit and false alarm rates in Figure 3B. The functions converge at $0^{\circ}$ azimuth (safe trials) because individual criteria were adjusted to produce false alarm rates near $16 \%$. The criteria ranged from a bin count of 10-17. Most mice produced a steeply sloped psychometric function that approached a hit rate of $100 \%$ at lateralized speaker locations.

The hit and false alarm rates in Figure 3B have been translated to the $d^{\prime}$ index of discrimination in Figure 3C. The discrimination functions recapitulate the relative performance of individual subjects that was captured by the psychometric functions in Figure 3B.

MAAs were interpolated from the discrimination functions as the speaker location that produced $d^{\prime}=1$. These thresholds ranged from $24^{\circ}$ to $37^{\circ}$ and averaged $31^{\circ}( \pm 5.9 \mathrm{SD})$, which corresponds well with thresholds that have been previously measured in other laboratory strains with similar psychophysical procedures (Heffner et al. 2001).

\section{Acoustic cues in the horizontal plane}

The acoustic basis of directional acuity in the horizontal plane is summarized by the HRTFs of two untrained CBA/129 mice in Figure 4. Both examples display similar directional effects. At central locations in the ipsilateral sound field, the HRTFs display sharp spectral notches. As the sound source moves to more lateralized locations, the low-frequency edge of the notches shifts to higher frequencies and decreases in depth. At highly lateralized locations, the overall gain of the HRTF decreases.

HRTFs in the contralateral sound field are marked by the directionally dependent gain changes that are generated by the sound shadow of the head. The magnitude of the shift grows with frequency to approximately $10 \mathrm{kHz}$, where a complex pattern of spectral notches creates a precipitous drop in the gain of the HRTF. Once again, the notches provide systematic frequency cues that are related to the direction of the sound source. More lateralized sources produce notch features at lower frequencies.

The effects of sound location on ILD and monaural spectral cues are summarized in Figure 5. ILD was calculated by subtracting the decibel gain of contralateral HRTFs from the complementary ipsilateral HRTFs (Fig. 5A). ILDs increased at more lateralized locations and showed larger disparities at higher frequencies. A sharp increase in ILD magnitude was produced at frequencies above $10 \mathrm{kHz}$ by

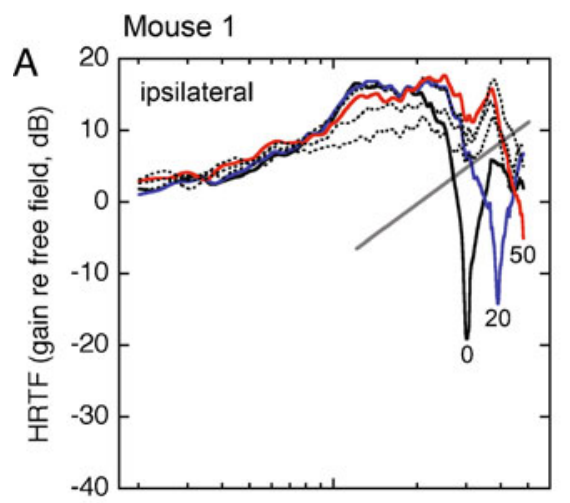

Mouse 2
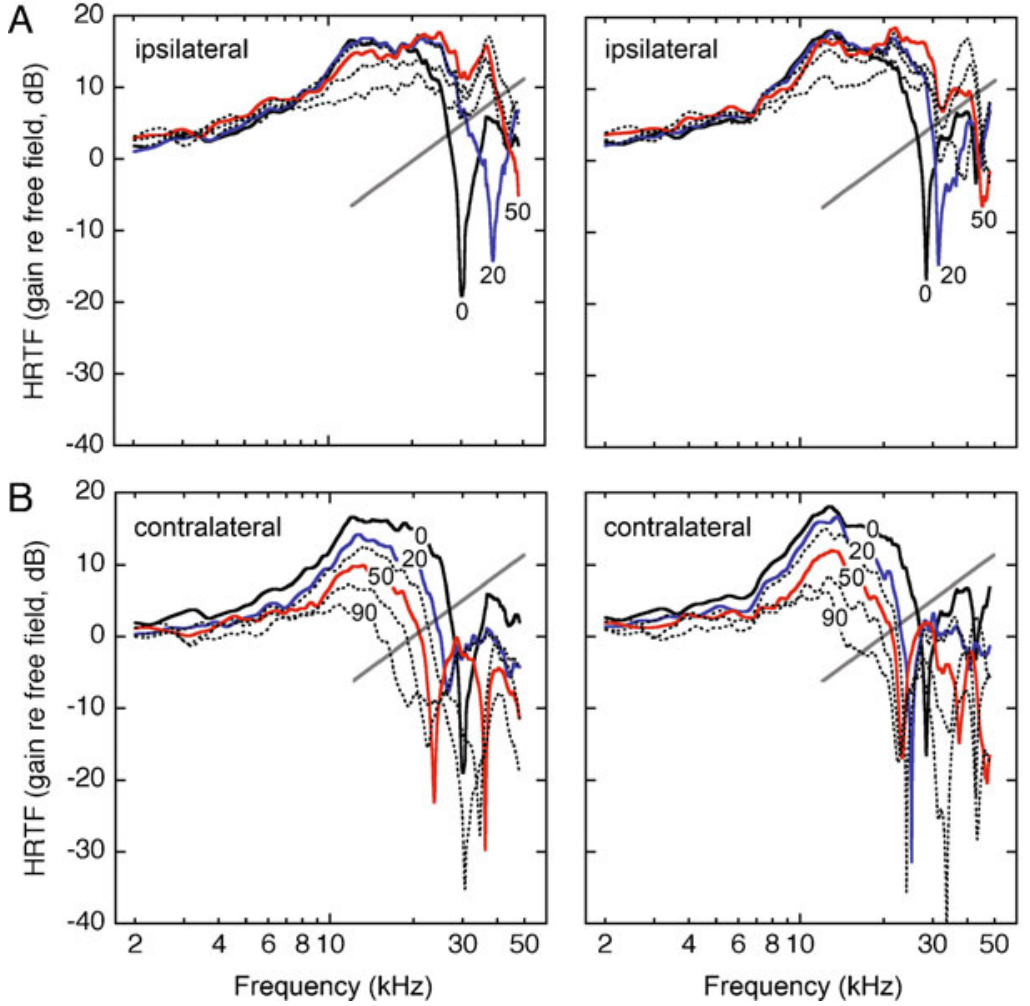

Mouse 2 (pinna removed)
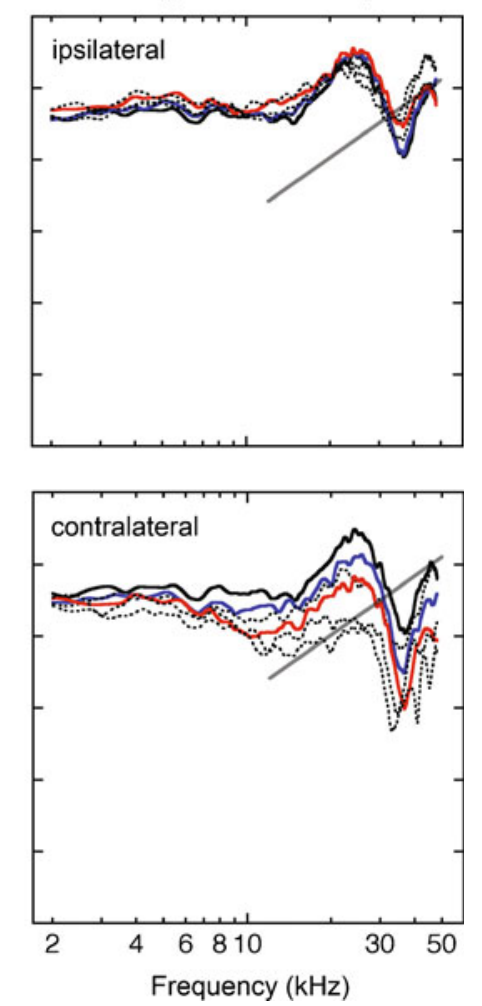

FIG. 4. Head-related transfer functions for 13 sound source locations in the inter-aural horizontal plane. Results from two mice are compared at complementary ipsilateral (A) and contralateral locations (B). The magnitude spectra are described in terms of decibel gain re free-field. Numerical labels indicate the azimuth of the sound source. The HRTFs for two ipsilateral and contralateral locations $\left(20^{\circ}\right.$ and $\left.50^{\circ}\right)$ are plotted in color to emphasize the systematic changes in gain and spectral shape. Gray lines highlight the systematic directional trends of the lowfrequency edge of spectral notches. The rightmost column shows the effects of pinna removal on the HRTFs of mouse 2. 


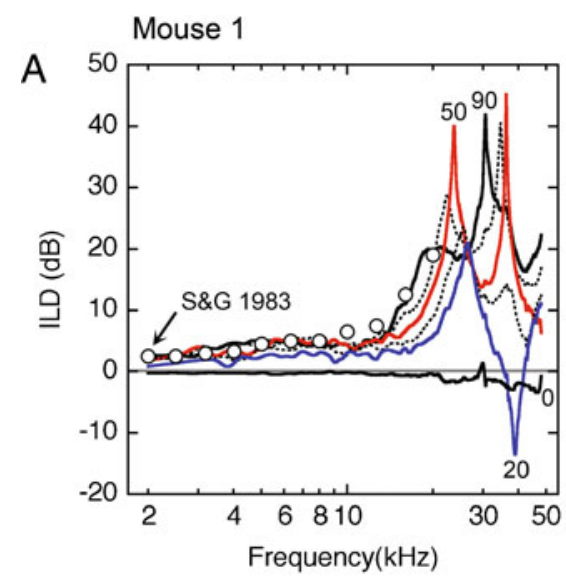

Mouse 2
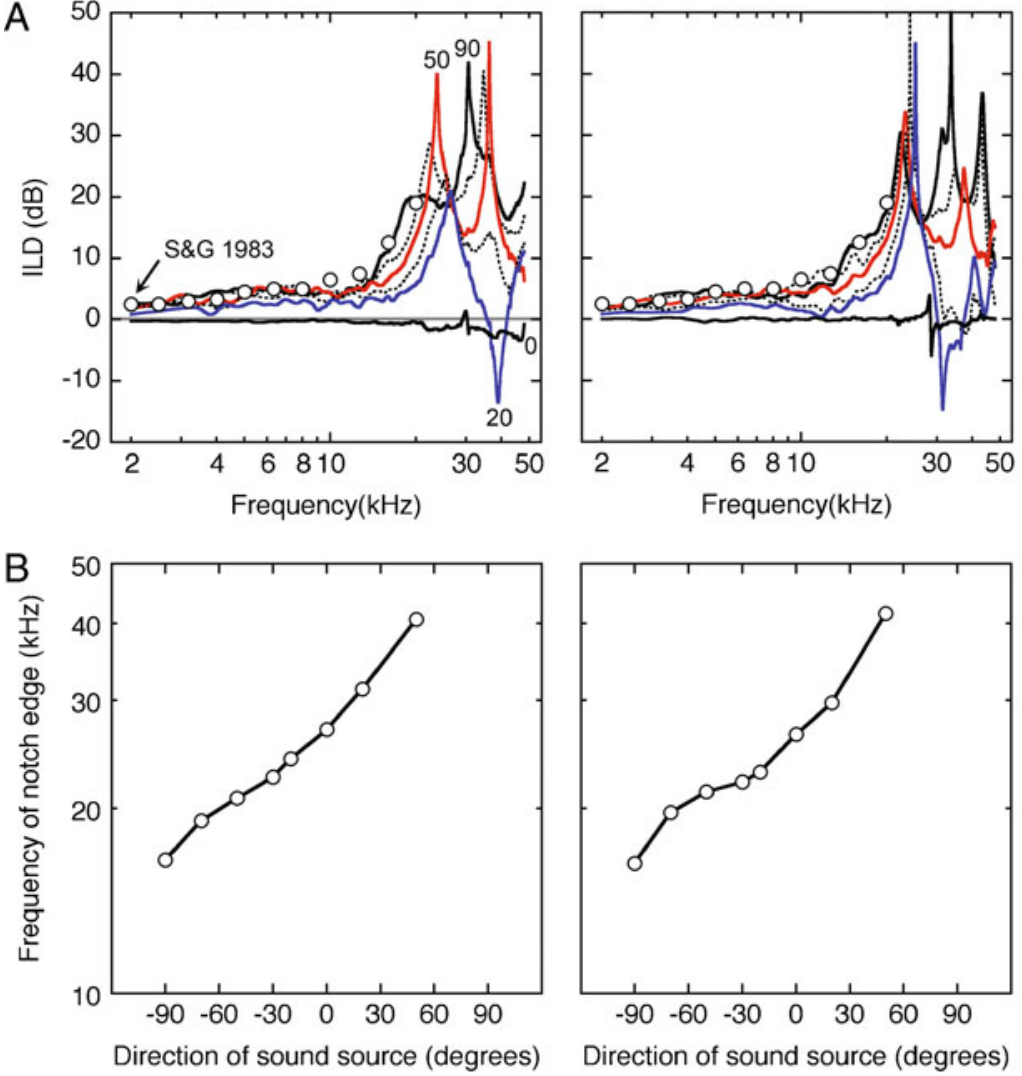

Mouse 2 (pinna removed)
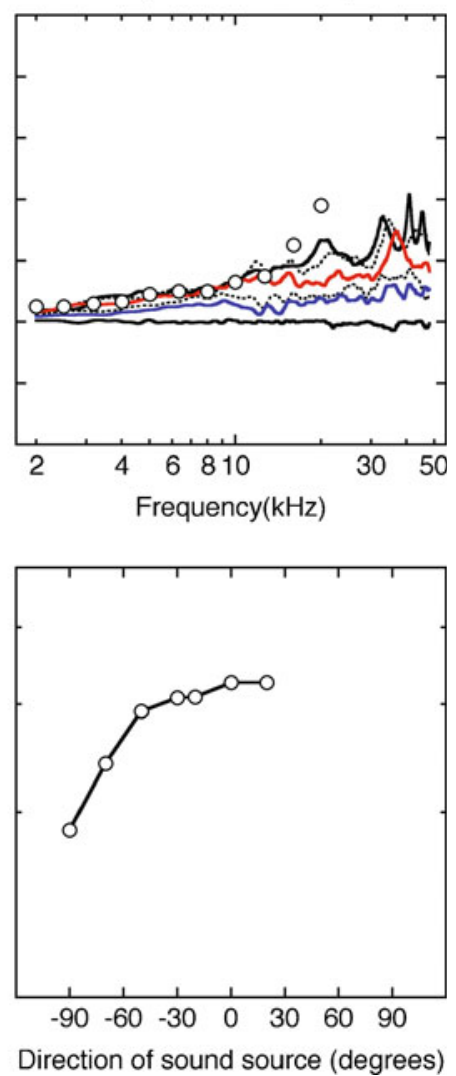

FIG. 5. Acoustic cues for directional acuity in the horizontal plane. Effects of azimuth on ILD (A) and the low-frequency edge of spectral notches (B). Results are shown for two mice. The rightmost column summarizes the effects of pinna removal on ILDs that were recorded

deep spectral notches in the contralateral HRTFs. Previously published pure tone data (Saunders and Garfinkle 1983) correspond well with current Golay measures at frequencies below $20 \mathrm{kHz}$.

Systematic changes in the low-frequency edge of spectral notches were illustrated by projecting an arbitrary line across the high-frequency notch region of the HRTFs and measuring the point of intersection (gray lines in Fig. 4). These results are shown in Figure 5B. Both mice showed a continuous increase in notch frequency as the sound source moved from lateralized locations in the contralateral hemifield to the opposite side of the head. Results in the ipsilateral hemifield are truncated at $50^{\circ}$ azimuth because edge frequencies converged on the upper limits of acoustic recordings. It is possible that extended sampling would reveal even higher edge frequencies at more lateralized ipsilateral azimuths.

Gross effects of the pinna on ILD and spectral features of the HRTF were illustrated by removing the outer ear during acoustic measurements in mouse 2. HRTFs showed a loss of mid-frequency gain and highfrequency notches after this manipulation (right column in Fig. 4A, B). As a result, the magnitude of from mouse 2. For comparison with previously published acoustic measures, each ILD plot presents pure tone data that were recorded at $90^{\circ}$ azimuth by Saunders and Garfinkle (1983) (open symbols).

the ILD was reduced at mid frequencies and spectral notches showed less directionality in the ipsilateral hemifield (right column in Fig. 5A, B).

\section{Manipulations of stimulus properties}

The acoustic features of horizontal HRTFs suggest multiple potential cues for spatial discrimination. At frequencies below $10 \mathrm{kHz}$, mice may attend to binaural ILD cues that are created by the geometric properties of the head. At frequencies above $10 \mathrm{kHz}$, ILD effects are sharply increased in magnitude by spectral notches. These directional properties of the pinna also create monaural spectral features that are useful in sound localization (Rice et al. 1992; Huang and May 1996). The relative importance of these three sources of directional information was first addressed by measuring how well mice discriminated changes in the horizontal location of band-limited noise that fell below or above $10 \mathrm{kHz}$.

The average discrimination functions for low-pass noise $(<10 \mathrm{kHz})$ and high-pass noise $(>10 \mathrm{kHz})$ are shown in Figure 6A. For comparison, the average discrimination function for broadband noise is also 
shown. Performance showed statistically significant improvements at more lateralized speaker locations, but was not influenced by frequency content $(p<0.05$, two-way ANOVA). Because HRTFs only displayed monaural spectral cues at frequencies above $10 \mathrm{kHz}$, the absence of a frequency effect suggests that ILD cues played a primary role in the discrimination of both low- and high-frequency sound sources.

Directional acuity can be attributed to the combined effects of enhanced ILD effects and spectral notches at frequencies above $10 \mathrm{kHz}$. Additional testing was conducted with pure tones to isolate the specific contribution of ILD information. The tones were frequency components of the low-pass and highpass noise that maintained the discrete ILD properties of the HRTF but lacked sufficient bandwidth to convey spectral features (Flannery and Butler 1981).

The localization of pure tones is summarized in Figure 6B. Average $d^{\prime}$ scores are presented for four frequencies at a fixed speaker location of $90^{\circ}$. The discrimination of band-limited noise is also shown for the same location. Relative to results that were obtained with band-limited noise of similar frequency content, the mice showed a consistent decline in discrimination when tested with pure tones. The deficit was statistically significant when $12-$ and $16-\mathrm{kHz}$ tones were compared with high-pass noise $(p<0.05$, oneway ANOVA, implemented in MATLAB using the anoval function), but not when 4 - and $8-\mathrm{kHz}$ tones were compared with low-pass noise. These results suggest that bandwidth plays an important role in directional acuity at frequencies above $10 \mathrm{kHz}$, but not at frequencies below $10 \mathrm{kHz}$. The bandwidth advantage may be gained from the availability of HRTF-based spectral cues (Fig. 4) or from the disambiguation of directional filtering effects that may create unusual ILD relationships at high frequencies (Fig. 5A).

\section{Manipulations of pinna acoustics}

Additional behavioral experiments investigated the importance of ILD and spectral cues by plugging the ear canal or distorting the shape of the pinna. The ear plugging procedure modified ILD by introducing a unilateral conductive loss. Pinna distortion altered HRTF-based spectral cues.

For this analysis, localization deficits were measured with broadband noise in the horizontal plane. Results are reported in terms of the absolute change in $d^{\prime}$ relative to each subject's performance under normal listening conditions (Fig. 3C). The comparisons were limited to locations where mice showed accurate baseline performance without pinna manipulations $\left(50^{\circ}\right.$, $70^{\circ}$, and $90^{\circ}$ ).

Three patterns of discrimination errors were produced by pinna manipulations. As shown in Figure 7A, a large deficit was observed when the plug was placed in the ear nearest the speaker array (ipsilateral plug). Mouse M2009_MU showed a 2-3 $d^{\prime}$ loss of performance. A second mouse (M2009_PA) experienced complete behavioral disruption and contributed sufficient warning trials for only one test location $\left(70^{\circ}\right.$ azimuth). These robust deficits suggest that the ipsilateral plug corrupted binaural ILD cues by removing the natural loudness advantage of the near ear. Similarly, the concomitant pinna distortion degraded monaural spectral cues. Although monaural spectral cues in the far ear were not affected, they apparently failed to provide reliable directional information.

The opposite behavioral outcome was observed when the pinna of the far ear was bonded in a folded position (contralateral distortion). As shown in Figure 7D,
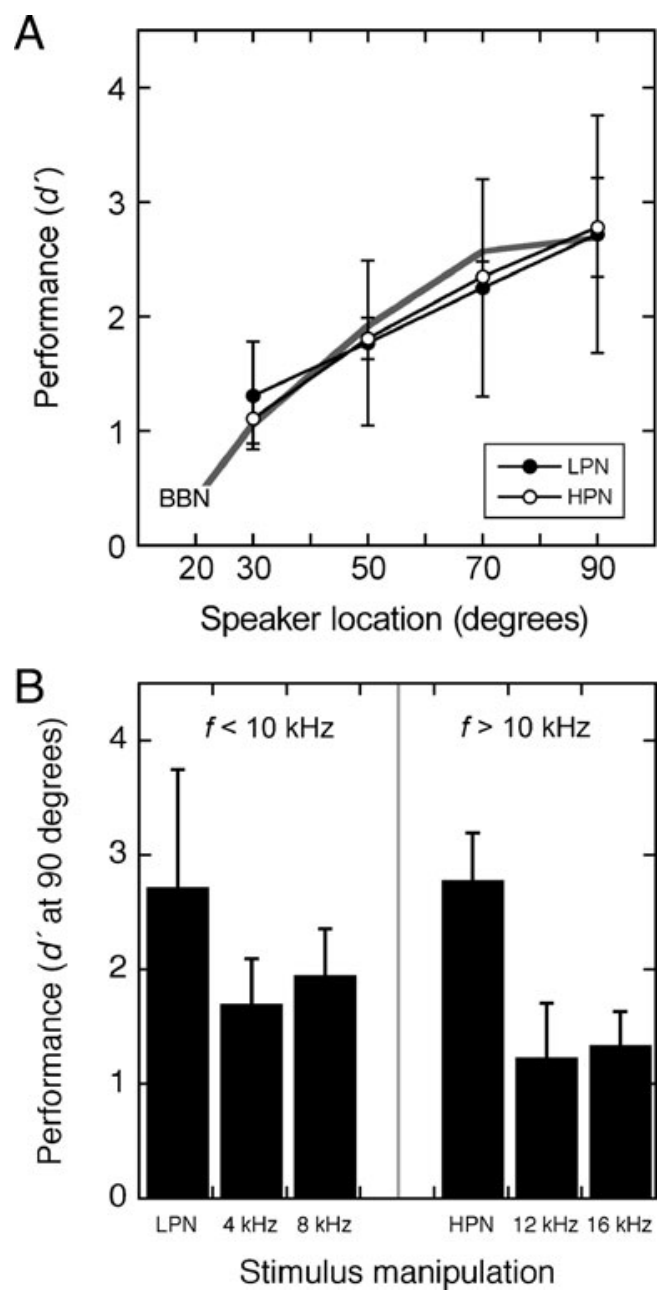

FIG. 6. Effects of stimulus manipulations on horizontal acuity. A Average $( \pm \mathrm{SD})$ discrimination functions for low-pass $(L P N)$ and highpass noise $(H P N)$ with cutoff frequencies of $10 \mathrm{kHz}$. Results are shown for four mice. For comparison, the average discrimination function of broadband noise $(B B N)$ is also shown for the same four mice. B Average $( \pm S D)$ discrimination scores for pure tones. Frequencies were selected to represent components of the LPN and HPN. Testing was only conducted at $90^{\circ}$. Results obtained at the same location with broadband noise have been extracted from $\mathbf{A}$. 

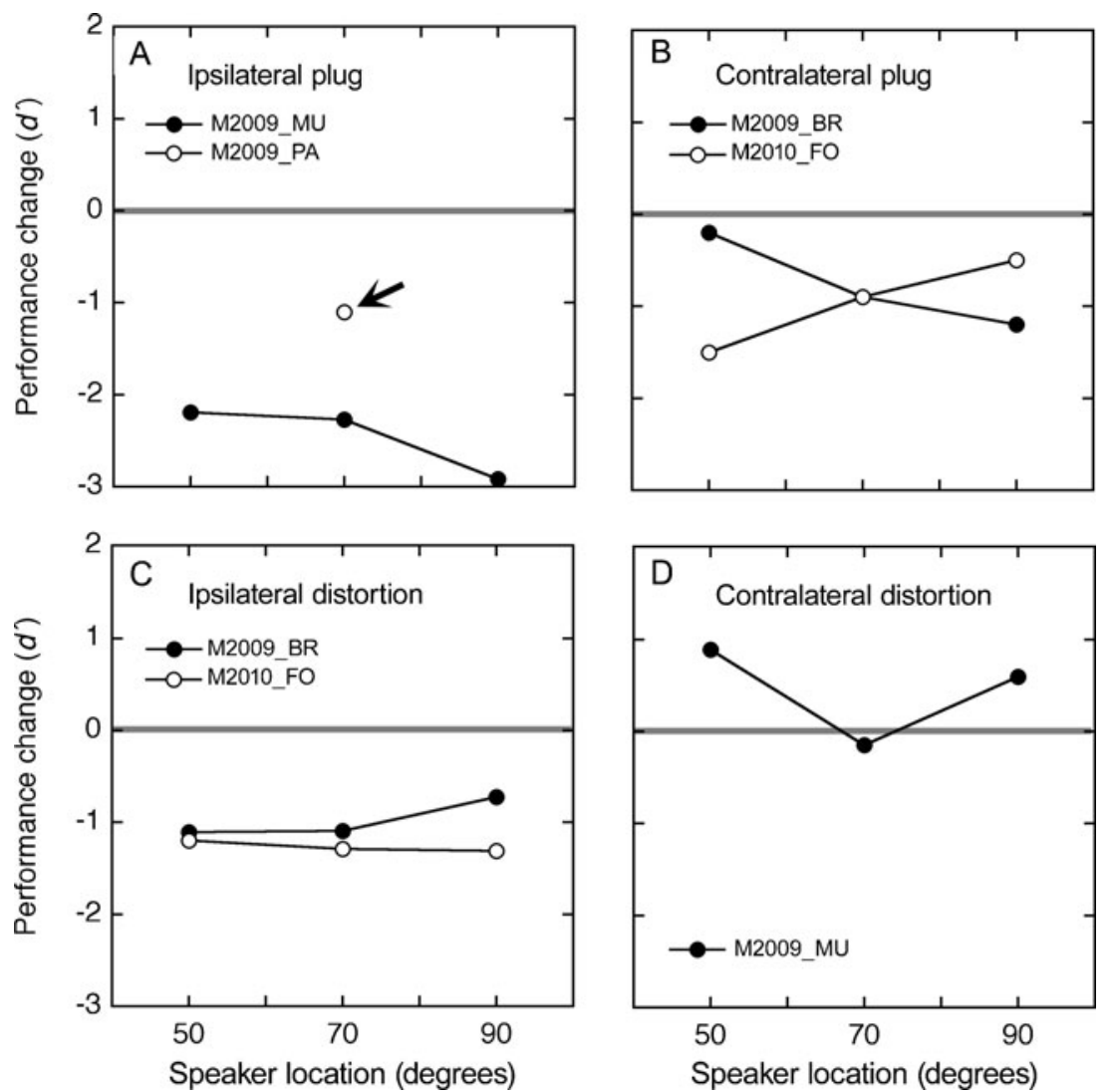

FIG. 7. Effects of pinna manipulations on horizontal acuity. Discrimination functions were measured in four mice with bursts of broadband noise while one ear was plugged (A, B) or distorted in shape (C, D). The manipulations were applied individually to the ear near the speaker array (ipsilateral) or the far ear (contralateral). Results are described in terms of the change in $d^{\prime}$ relative to normal performance. Arrow, mouse M2009_PA showed complete behavioral disruption when tested with an ipsilateral ear plug. Data from this mouse are shown for the only location that generated more than five trials. mouse M2009_MU produced $d^{\prime}$ scores that matched or exceeded its normal baselines. These findings provide further evidence for a weak contribution of contralateral spectral cues to spatial discrimination. Presumably, the mice maintained accurate localization behavior by attending to binaural ILD cues or ipsilateral spectral cues. Neither source of directional information was severely compromised by folding the contralateral ear.

Intermediate behavioral deficits were produced by a contralateral ear plug (Fig. 7B) or ipsilateral pinna distortion (Fig. 7C). The former manipulation disrupted binaural ILD cues but preserved spectral cues in the near ear, while the latter had the opposite effect. The incomplete loss of directional acuity under both conditions suggests that mice were able to maintain discrimination during less extreme modifications of binaural ILD cues (ipsilateral distortion) or when monaural spectral cues remained intact in the near ear (contralateral plug).

\section{Directional acuity in the median vertical plane}

The spatial discrimination of sound sources in the median vertical plane proved to be a difficult task for the mice. To avoid behavioral disruption, testing was limited to broadband noise and the range of comparison speaker locations was reduced to $30^{\circ}, 50^{\circ}, 70^{\circ}$, and $90^{\circ}$.
Despite these measures, one mouse (M2010_PE) failed to attain stable performance and was excluded from the following analysis. The remaining four mice completed at least 600 safe trials and 91 warning trials.

The individual bin counts of the four mice are shown in Figure 8A. On average, pre-stimulus counts (17.2 \pm 0.8 SD) did not change relative to test results from the same mice in the horizontal plane $(17.1 \pm 1.0 \mathrm{SD} ; N S$, paired $t$ test implemented in MATLAB using the ttest function). Peri-stimulus bin counts for safe trials were also unaffected $(17.3 \pm 1.4 \mathrm{SD}$, vertical; $17.6 \pm$ $1.0 \mathrm{SD}$, horizontal). In contrast, peri-stimulus bin counts for warning trials failed to attain the levels of suppression that were observed for testing with broadband noise in the horizontal plane (Fig. 3A). Nevertheless, because a small but consistent suppression was observed for more lateralized speaker placements, the effect of location remained statistically significant (two-way ANOVA, $p<0.05$ ).

The average hit rates and $d^{\prime}$ scores for locations in the median vertical plane are shown in Figure 7B, C, respectively. Because the criterion for suppression was based on each subject's responses to broadband noise in the horizontal plane, the false alarm rates are no longer locked to $16 \%$. The small deviations from this value in Figure $8 \mathrm{~B}$ further confirm that the mice produced similar bin counts during safe trials under both stimulus manipulations. The shallow slopes of 
the functions reflect higher bin counts during warning trials (i.e., less suppression).

The $d^{\prime}$ index of discrimination is shown in Figure 8C. Maximum values at locations of $70^{\circ}$ and $90^{\circ}$ fall close to the threshold for discrimination. As a result, three subjects produced MAAs that averaged 80.7 ( $\pm 1.7 \mathrm{SD})$ degrees. The remaining subject (M2009_MU) failed to reach the threshold criterion.
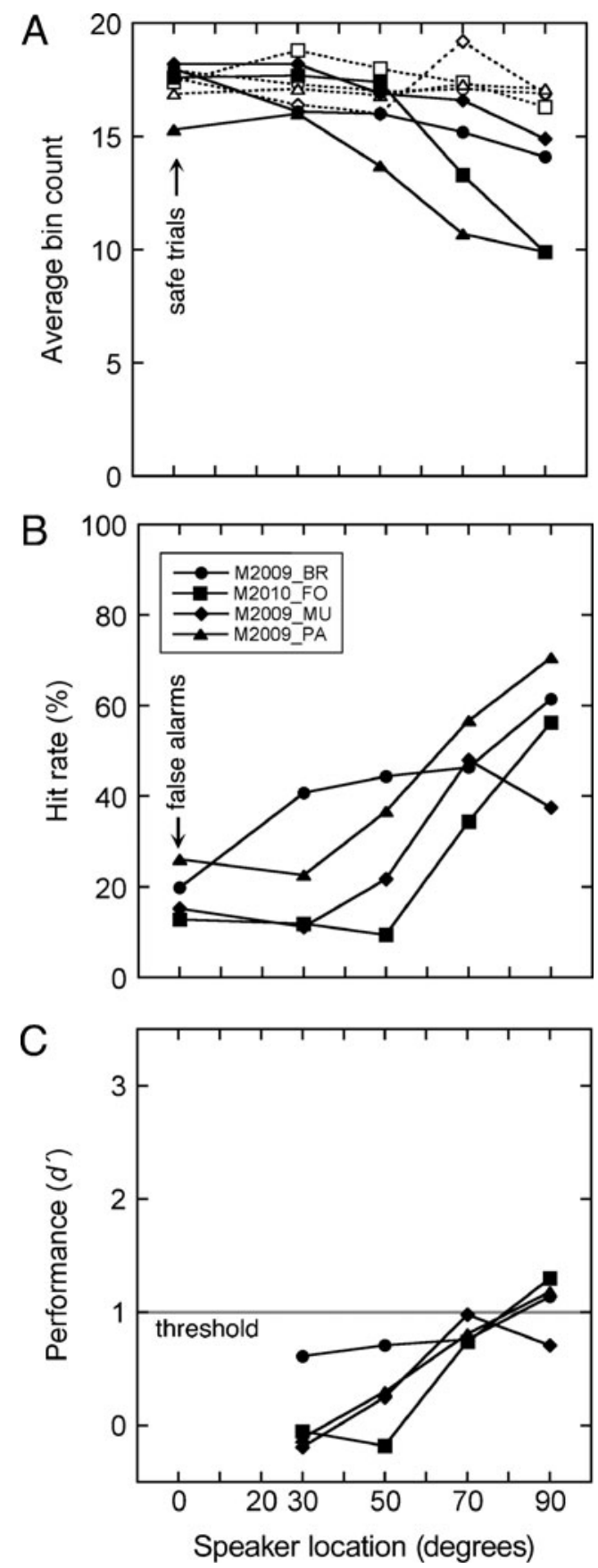

FIG. 8. Directional acuity for presentations of broadband noise in the median vertical plane. Results are shown for four mice. Plotting conventions are described in Figure 3.
Acoustic cues in the median plane

HRTFs for six elevations $\left(0^{\circ}, 20^{\circ}, 30^{\circ}, 50^{\circ}, 70^{\circ}\right.$, and $90^{\circ}$ ) in the median vertical plane are compared in Figure 9. The acoustic measurements were virtually identical at frequencies below $10 \mathrm{kHz}$. Directionally dependent gain differences remained subtle at frequencies between 10 and $20 \mathrm{kHz}$, although both mice displayed a loss of gain when sounds were delivered from an elevation of $90^{\circ}$ (i.e., directly over the head). Spectral notches were visible at frequencies above $20 \mathrm{kHz}$. Unlike spectral cues for azimuth, the lowfrequency edge of these spectral notches did not change systematically with source location.

The role of the pinna in the production of spectral cues for elevation was examined by recording HRTFs in the median plane after the pinna was removed from mouse 2 (right column in Fig. 9). As predicted by the well-established role of the pinna in vertical spatial acuity (Gardner and Gardner 1973; Wightman and Kistler 1989b; Butler and Humanski 1992), directional differences were negligible across the entire range of sampled frequencies after this manipulation. Gain modulations above $20 \mathrm{kHz}$ were produced by non-directional resonances in the intact cavities of the outer ear and ear canal (Shaw 1974).

\section{DISCUSSION}

CBA/129 mice displayed their best auditory spatial acuity when localizing the multiple directional cues that are conveyed by broadband noise in the inter-aural horizontal plane. The average MAA of five mice was $31^{\circ}$ (Fig. 3). One other study using similar psychoacoustic methods has reported an average threshold of $33^{\circ}$ for young C57BL/6J mice (Heffner et al. 2001). The close agreement of localization performance for these genetically diverse subject groups suggests that the general mechanisms of directional hearing are conserved in common strains of laboratory mice.

\section{Alternative measures of directional hearing in laboratory mice}

As an alternative to the intensive testing that is required by conventional psychophysical methods, prepulse inhibition (PPI) is gaining popularity as a metric for assessing auditory behavior in mice. PPI refers to a decrease in the acoustic startle reflex that is observed when a detectable acoustic event (the prepulse) is delivered immediately before the startle-eliciting stimulus. In the context of directional hearing, a sound source shifts from one speaker location to another. The discriminability of the change in location is reflected in the magnitude of PPI. 


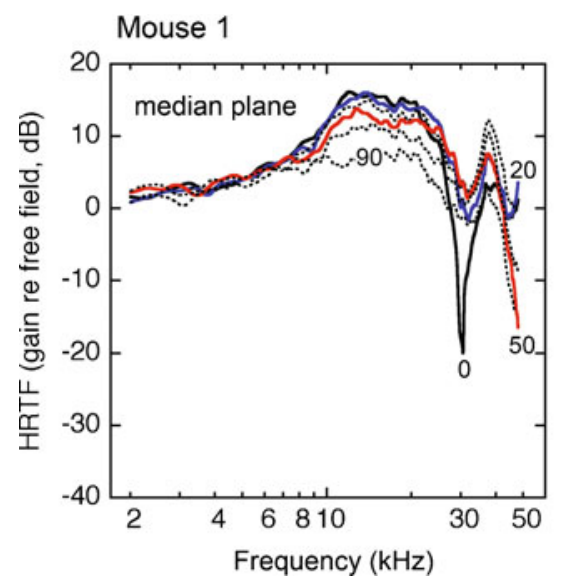

Mouse 2

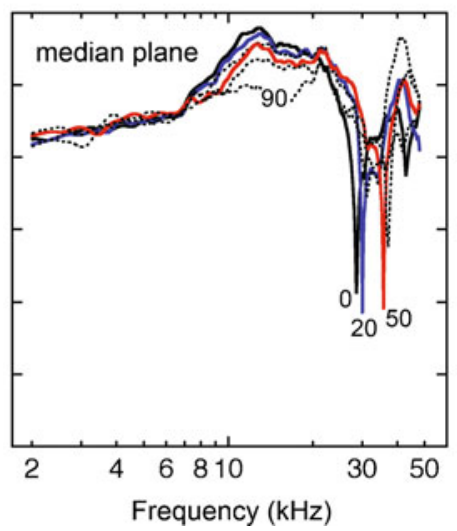

Mouse 2 (pinna removed)

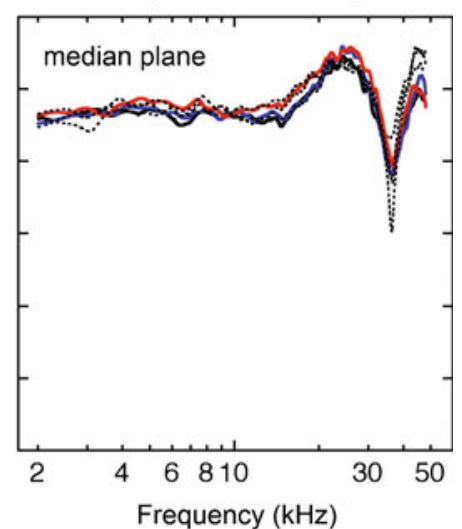

FIG. 9. Head-related transfer functions for six sound source locations in the median vertical plane. Results are shown for two mice. Recordings were made from mouse 2 before and after the pinna was surgically removed. Additional plotting conventions are described in Figure 4.

The "speaker swap" procedure has been used to measure the horizontal spatial acuity of CBA/CaJ mice (Allen and Ison 2010). In that study, broadband noise elicited PPI at angles of azimuth as small as $15^{\circ}$. At present, it is not clear how the magnitude of PPI translates to conventional psychophysical measures of hit rate or $d^{\prime}$ that were obtained in the present study. For example, CBA/129 produced positive detection scores at angles of $20^{\circ}$ (Fig. 3), but this performance fell below standardized threshold criteria. Although the relationship between PPI and directional hearing requires further analysis, the speaker swap paradigm produces a well-controlled perceptual effect that has important implications for future studies of directional acuity in laboratory mice.

Existing studies of directional hearing in laboratory mice have focused on MAAs, which are based on the subject's ability to detect relative directional changes between multiple sound sources. Absolute localization (i.e., the ability to locate a discrete sound source in absolute spatial coordinates) has been measured with a goal orientation paradigm (Ehret and Dreyer 1984). In that study, NMRI mice obtained water rewards by approaching a sound source that signaled the location of an active water spout. Navigational errors were $<15^{\circ}$ when the target location was indicated by a continuous train of tone bursts (closed-loop paradigm). Mice were unable to perform the task when the tone bursts were turned off before the initiation of the search behavior (open-loop paradigm).

The superior localization of mice in the closed-loop paradigm is expected given the availability of nondirectional cues, such as the loudness effects that are generated by head movements or increased proximity to the sound source. Difficulty with the open-loop paradigm is predicted by previous studies of rodent species. Although cats produce localization errors of $<10^{\circ}$ when tested under open-loop conditions (Casseday and Neff 1973), laboratory rats fail to discriminate two sources that are placed in the same hemifield (Kavanagh and Kelly 1986).

\section{ITDs in laboratory mice}

The mammalian auditory system maintains its exquisite directional acuity in the horizontal plane by transitioning between low-frequency inter-aural time differences (ITD) cues and high-frequency ILD cues (Strutt 1907; Mills 1958). The relative importance of this duplex mode of directional hearing has been previously evaluated in mice by measuring localization performance during manipulations of stimulus bandwidth and frequency. It has been shown that C57BL/ $6 \mathrm{~J}$ mice cannot localize band-pass noise in the interaural horizontal plane when spectral energy is limited to frequencies below $10 \mathrm{kHz}$ (Heffner et al. 2001; Allen and Ison 2010). This deficit has led to the conclusion that laboratory strains do not make effective use of low-frequency ITD cues.

The binaural processing of ITD information is further constrained in mice by poor hearing sensitivity at frequencies below $4 \mathrm{kHz}$, where other mammalian species are most sensitive to ITD cues (Radziwon et al. 2009). Recent electrophysiological studies of laboratory mice report a comparable upper limit of phase locking in the auditory nerve (Taberner and Liberman 2005). Because the degree of monaural synchronization tends to be lower than in mammalian species with lowfrequency hearing (e.g., the domestic cat), these temporal response patterns are less likely to support binaural ITD sensitivity.

Brainstem structures for processing low-frequency temporal information are also poorly developed. The medial superior olive of the mouse contains only 210 projection neurons, where over 4200 neurons are found in the domestic cat (Irving and Harrison 1967). Species differences are less apparent for the primary processing centers of high-frequency ILD cues. The 
medial nucleus of the trapezoid body and lateral superior olive contain approximately 2,270 and 1,190 neurons in the mouse in comparison to 5,910 and 3,360 neurons in the cat, respectively (Irving and Harrison 1967).

Although published studies have reported pronounced localization deficits at frequencies below $10 \mathrm{kHz}$ (Heffner et al. 2001; Allen and Ison 2010), CBA/129 mice performed well under similar conditions in the present study. Low-pass noise produced discrimination functions that were equivalent to both broadband noise and high-pass noise (Fig. 6A). Tonal frequencies produced discrimination scores that surpassed the criterion for threshold performance at a speaker location of $90^{\circ}$ (Fig. 6B). Acoustic data confirmed the availability of ILD cues to support this discrimination (Fig. 5A). Although the contributing factors for this apparent discrepancy remain unknown, recent studies of $\mathrm{CBA} / \mathrm{CaJ}$ mice replicate present findings by demonstrating a slight enhancement in the localization of low-pass noise (June et al. 2010).

\section{Role of monaural spectral cues}

The HRTF of the mouse displays a complex pattern of peaks and notches that varies with source locations in the inter-aural horizontal plane (Fig. 4) and median vertical plane (Fig. 9). These directionally dependent features were most apparent at central sound locations (i.e., directly in front of the subject's head). At increasing ipsilateral azimuths and elevations, the HRTF was devoid of sharp spectral features and instead displayed a progressive decrease that was more uniformly distributed across frequency. The presence of notches at contralateral azimuths contributed to a steep rise in the ILD at frequencies above $10 \mathrm{kHz}$ (Fig. 5A). Monaural spectral cues were created by the effect of azimuth on the low-frequency edge of spectral notches (Fig. 5B).

Although global changes in HRTF gain were correlated with both horizontal and vertical sound locations, spatial acuity was better in the horizontal plane. The sharp contrast in performance suggests that the mice were able to derive directionality from the ILD effect that was generated at eccentric azimuths, but not from the sequential level disparities that were produced by changes in elevation. The natural relevance of these cues may be underestimated by the present paradigm, however, because inter-speaker level differences were intentionally made ambiguous by roving stimulus level.

The role of monaural spectral cues was also investigated by altering the acoustic properties of the outer ear. Surgical removal of the pinna produced an HRTF that remained largely unaffected by changes in elevation (right column in Fig. 9). Occlusion of human pinna cavities is known to increase errors in vertical localization and front-back confusions (Gardner and Gardner 1973). Similar deficits are observed when localization is simulated under closed-field conditions if pinna-based spectral cues are poorly synthesized (Wightman and Kistler 1989a, b) or are based on non-individualized transfer functions (Wenzel et al. 1993; Van Wanrooij and Van Opstal 2005). These results suggest that generalized ILD cues are sufficient for deriving source azimuth, but individualized spectral cues are necessary for determining elevation. Consequently, the poor elevation sensitivity of CBA/129 mice may reflect inadequate spectral cues or the absence of brainstem mechanisms that are known to process this information.

Neurophysiological studies in other mammalian species have identified neural populations that perform wideband spectral integration in the dorsal cochlear nucleus (DCN; Young et al. 1992; Imig et al. 2000) and the central nucleus of the inferior colliculus (ICC; Davis et al. 2003; Chase and Young 2005). Surgical lesions of this spectral processing pathway produce localization deficits that are most obvious for the identification of sound source elevation (Sutherland et al. 1998; May 2000). It is intriguing that single-unit studies of the DCN and ICC of laboratory mice have noted a striking underrepresentation of those specialized neural response patterns (Ma et al. 2006; Ma and Brenowitz 2010). Based on our present behavioral findings, this "natural ablation" of the spectral processing pathway reproduces the deleterious effects of DCN lesions on vertical sound localization.

Relative to well-established models of mammalian hearing such as the domestic cat, the few existing studies of laboratory mice demonstrate a consistent lack of directional acuity, a limited availability of veridical localization cues, and an absence of specialized pathways for the auditory processing of ITD and pinna-based spectral information. In combination, these results provide further evidence for a strong dichotomy in the auditory perceptual abilities of common prey and predatory species (Heffner and Heffner 1988).

\section{ACKNOWLEDGMENTS}

This work was supported by NIH grant DC005211 and intramural funding from the Department of OtolaryngologyHead and Neck Surgery, The Johns Hopkins University School of Medicine.

\section{CONFLICT OF INTEREST}

The authors have no commercial interest or conflict of interest to disclose. 


\section{REFERENCES}

Allen PD, Ison JR (2010) Sensitivity of the mouse to changes in azimuthal sound location: angular separation, spectral composition, and sound level. Behav Neurosci 124:265-277

ButLer RA, Humanski RA (1992) Localization of sound in the vertical plane with and without high-frequency spectral cues. Percept Psychophys 51:182-186

Casseday JH, NefF WD (1973) Localization of pure tones. J Acoust Soc Am 54:365-372

Chase SM, Young ED (2005) Limited segregation of different types of sound localization information among classes of units in the inferior colliculus. J Neurosci 25:7575-7585

Chen QC, Cain D, Jen PH (1995) Sound pressure transformation at the pinna of Mus domesticus. J Exp Biol 198:2007-2023

Davis KA, Ramachandran R, May BJ (2003) Auditory processing of spectral cues for sound localization in the inferior colliculus. J Assoc Res Otolaryngol 4:148-163

Ehret G, Dreyer A (1984) Localization of tones and noise in the horizontal plane by unrestrained house mice (Mus musculus). J Exp Biol 109:163-174

FlanNery R, ButLer RA (1981) Spectral cues provided by the pinna for monaural localization in the horizontal plane. Percept Psychophys 29:438-444

Gardner MB, Gardner RS (1973) Problem of localization in the median plane: effect of pinnae cavity occlusion. J Acoust Soc Am 53:400-408

HefFner RS, HefFner HE (1988) Sound localization in a predatory rodent, the northern grasshopper mouse (Onychomys leucogaster). J Comp Psychol 102:66-71

Heffner RS, Koay G, Heffner HE (2001) Sound localization acuity changes with age in C57BL.6J mice. In: Willott JF (ed) Handbook of mouse auditory research: from behavior to molecular biology. CRC, Boca Raton, pp 31-36

Huang AY, MaY BJ (1996) Sound orientation behavior in cats. II. Mid-frequency spectral cues for sound localization. J Acoust Soc Am 100:1070-1080

Imig TJ, Bibikov NG, Poirier P, SAmson FK (2000) Directionality derived from pinna-cue spectral notches in cat dorsal cochlear nucleus. J Neurophysiol 83:907-925

Irving R, HARRISON JM (1967) The superior olivary complex and audition: a comparative study. J Comp Neurol 130:77-86

June KM, Radziwon Ke, Xu-Friedman MA, Salvi RJ, Dent ML (2010) Sound localization acuity in the CBA/CaJ mouse (Mus musculus). Abstr Assoc Res Otolaryngol 33:311

Kavanagh GL, Kelly JB (1986) Midline and lateral field sound localization in the albino rat (Rattus norvegicus). Behav Neurosci 100:200-205

MA W, BRENowitz S (2010) Single neuron recordings from unanesthetized mouse dorsal cochlear nucleus. Abstr Assoc Res Otolaryngol 34:429
Ma WL, Hidaka H, MaY BJ (2006) Spontaneous activity in the inferior colliculus of $\mathrm{CBA} / \mathrm{J}$ mice after manipulations that induce tinnitus. Hear Res 212:9-21

MAsterton RB (1997) Neurobehavioral studies of the central auditory system. Ann Otol Rhinol Laryngol Suppl 168:31-34

MAY BJ (2000) Role of the dorsal cochlear nucleus in the sound localization behavior of cats. Hear Res 148:74-87

Mills AW (1958) On the minimum audible angle. J Acoust Soc Am 30:237-246

NefF WD, CAsseday JH (1977) Effects of unilateral ablation of auditory cortex on monaural cat's ability to localize sound. J Neurophysiol 40:44-52

Radziwon Ke, June KM, Stolzberg DJ, Xu-Friedman ma, Salvi RJ, DENT ML (2009) Behaviorally measured audiograms and gap detection thresholds in CBA/CaJ mice. J Comp Physiol A Neuroethol Sens Neural Behav Physiol 195:961-969

Rice JJ, May BJ, Spirou GA, Young ED (1992) Pinna-based spectral cues for sound localization in cat. Hear Res 58:132-152

Saunders JC, GarfinkLe TJ (1983) Peripheral anatomy and physiology. In: Willott JF (ed) The auditory psychobiology of the mouse. Thomas, Springfield, pp 131-168

SHaw EAG (1974) The external ear. In: Keidel WD, Neff WD (eds) Handbook of sensory physiology. Springer, Berlin, pp 455-490

SleE SJ, Young ED (2010) Sound localization cues in the marmoset monkey. Hear Res 260:96-108

Strutt JW (1907) On the perception of sound direction. Phil Mag 13:214-232

Sutherland DP, Masterton RB, Glendenning KK (1998) Role of acoustic striae in hearing: reflexive responses to elevated soundsources. Behav Brain Res 97:1-12

Taberner AM, Liberman MC (2005) Response properties of single auditory nerve fibers in the mouse. J Neurophysiol 93:557569

Van Wanrooij MM, Van Opstal AJ (2005) Relearning sound localization with a new ear. J Neurosci 25:5413-5424

Wenzel EM, Arruda M, Kistler DJ, Wightman FL (1993) Localization using nonindividualized head-related transfer functions. J Acoust Soc Am 94:111-123

Wightman FL, Kistler DJ (1989A) Headphone simulation of freefield listening. I: Stimulus synthesis. J Acoust Soc Am 85:858-867

Wightman FL, KistLer DJ (1989в) Headphone simulation of freefield listening. II: Psychophysical validation. J Acoust Soc Am 85:868-878

Yoshida N, Hequembourg SJ, Atencio CA, Rosowski JJ, Liberman MC (2000) Acoustic injury in mice: $129 / \mathrm{SvEv}$ is exceptionally resistant to noise-induced hearing loss. Hear Res 141:97-106

Young ED, Sirou GA, Rice JJ, Voigt HF (1992) Neural organization and responses to complex stimuli in the dorsal cochlear nucleus. Philos Trans R Soc Lond B Biol Sci 336:407-413

Zhou B, Green DM, Middlebrooks JC (1992) Characterization of external ear impulse responses using Golay codes. J Acoust Soc Am 92:1169-1171 